\title{
The Principle of Limiting Amplitude for Symmetric Hyperbolic Systems in an Exterior Domain
}

\author{
By \\ Kiyoshi MochizUKI*
}

This paper is a supplement to the previous paper [2], in which we have treated the eigenfunction expansions and the scattering theory for symmetric hyperbolic systems in an exterior domain. The notation and the terminology of the preceding paper will be used freely.

Let $\partial G$ be a bounded closed hypersurface of class $C^{2}$ in $\boldsymbol{R}^{n}(n \geq 2)$, and $G$ the domain exterior to $\partial G$. Consider the following mixed initial-boundary value problem for hyperbolic system.

$$
\left\{\begin{array}{c}
\left\{M(x) \frac{\partial}{\partial t}-\sum_{j=1}^{n} A_{j}(x) \frac{\partial}{\partial x_{j}}-B(x)\right\} u(x, t)=g(x) e^{i \mu t} \quad \text { for } t>0, x \in G ; \\
u(x, 0)=u_{0}(x) \quad \text { for } x \in G ; \\
u(z, t) \in N(z) \quad \text { for } t>0, z \in \partial G .
\end{array}\right.
$$

Here $\mu(\neq 0)$ is a real number, $i=\sqrt{-1}, u(x, t)$ and $g(x)$ are vector valued functions whose values lie in $\boldsymbol{C}^{m}$, and $M(x)$ (measurable), $A_{j}(x)$ (smooth) and $B(x)$ (continuous) are $m \times m$ matrix valued, bounded functions. The boundary space $N(z)$ is a linear subspace of $\boldsymbol{C}^{m}$ of constant dimension which is smoothly varying on $\partial G$. Our aim is to derive the limiting amplitude principle; that is, we shall obtain the asymptotic behavior of solutions of the above problem.

The assumptions which we require below are summarized as

( I ) $M(x)$ is positive definite. $L=\frac{1}{i}\left\{\sum_{j=1}^{n} A_{j}(x) \frac{\partial}{\partial x_{j}}+B(x)\right\}$ is formally self-adjoint in $\mathscr{H}=\left[L^{2}(G)\right]^{m}$.

Received July 7, 1969.

Communicated by S. Matsuura.

* Yoshida College, Kyoto University. 
(II) For $|x|>\rho$ (large) $M(x)=I, A_{j}(x)=A_{j}$ (constant) and $B(x)=0$.

(III) $A(x, \xi)=\sum_{j=1}^{n} A_{j}(x) \xi_{j}$ is non-singular for each $x \in G$ and $\xi \in \boldsymbol{R}^{n}-\{0\}$, and $A^{0}(\xi)=\sum_{j=1}^{n} A_{j} \xi_{j}$ is isotropic :

$\operatorname{det}\left[A^{0}(\xi)-\lambda I\right]=\prod_{\nu=1}^{k}\left(\tau_{\nu}|\xi|-\lambda\right)^{m_{\nu}}, \sum_{\nu=1}^{k} m_{\nu}=m, \quad \tau_{1}>\tau_{2}>\cdots>\tau_{k}$.

(IV) $N(z)$ is maximally conservative with respect to $L$ at each point $z \in \partial G$, and is coercive for $L$ in the sense of Aronszajn (see [1], VI, Definition 2.1).

Remark 1. The above assumptions are stronger than those supposed in [2] since we require that $A(x, \xi)$ is non singular. This with the coercivity of $N(z)$ derives the Hölder continuity of the spectral density for $L_{1}=M^{-1}(x) L$ ([2]; Theorem 4.3), which plays an important role in our proof.

We denote by $\mathscr{H}_{1}$ the Hilbert space of square integrable functions in $G$ with norm

$$
\|f\|_{1}=\left\{\int_{G} M(x) f(x) \cdot \overline{f(x)} d x\right\}^{1 / 2}
$$

which is equivalent to the usual $L^{2}$-norm. Consider the problem (1) in $\mathscr{H}_{1}$. Under the above assumptions, the differential operator $\frac{1}{i} M^{-1}(x)\left\{\sum_{j=1}^{n} A_{j}(x) \frac{\partial}{\partial x_{j}}+B(x)\right\}$ uniquely determines a selfadjoint operator $L_{1}$ in $\mathscr{H}_{1}$. Thus the problem (1) with $g, u_{0}$ in $\mathscr{H}_{1}$ can be written in the following form:

$$
\frac{1}{i} \frac{\partial u}{\partial t}-L_{1} u=\frac{1}{i} M^{-1}(x) g(x) e^{i \mu t}, \quad u(x, 0)=u_{0}(x) .
$$

It has been shown in $\$ 4$ of [2] that under the above assumptions the point spectrum $\sigma_{p}\left(L_{1}\right)$ of $L_{1}$ consists of isolated eigenvalues which are of finite multiplicity except the origin 0 . Further, all eigenfunctions corresponding to non-zero eigenvalues are of bounded supports contained in the ball $\{|x|<\rho\}$. Thus, if the differential operator $L-\sigma M(x)$ has the unique continuation property, then $L_{1}$ has no eigenvalue except the origin 0 . We should remark, however, that the origin 0 is somewhat ambiguous; the null space $\Omega\left(L_{1}\right)$ of $L_{1}$ may or may not be empty, and if the latter is the case, then it 
remains as a problem whether the dimension of $\mathscr{I}\left(L_{1}\right)$ is finite or infinite.

We have derived also that if $\sigma$ (real) is not in $\sigma_{p}\left(L_{1}\right) \cup\{0\}$, then for any $f$ in $\mathscr{H}_{1}$ of bounded support there exists the limit $v_{-r}(\cdot, \sigma)$ as $\varepsilon \rightarrow \pm 0$ of $v(\cdot, \sigma+i \varepsilon)=\left(L_{1}-\sigma-i \varepsilon\right)^{-1} f$ satisfying the equation

$$
\left(L_{1, \mathrm{loc}}-\sigma\right) v_{+}=f
$$

and the radiation conditions at infinity (see Definition 4.1 of [2]). More precisely if we suppose that the interval $[a, b]$ does not contain any point of $\sigma_{p}\left(L_{1}\right) \cup\{0\}$, then $v(\cdot, \sigma+i \varepsilon)$ tends as $\varepsilon \rightarrow \pm 0$ in the sense of local energy norm to $v_{+}(\cdot, \sigma)$ uniformly with respect to $\sigma \in[a, b]$, and $v_{ \pm}(\cdot, \sigma)$ is Hölder continuous in $\sigma$ with exponent $h(0<h<1)$. Thus, if we denote by $E_{1}(\sigma)(-\infty<\sigma<\infty)$ the resolution of the identity associated with $L_{1}$, then it follows that

$$
\frac{d}{d \sigma} E_{1}(\sigma) f=\frac{1}{2 \pi i}\left\{v_{1}(\cdot, \sigma)-v_{-}(\cdot, \sigma)\right\} \quad \text { for } \sigma \in[a, b]
$$

and is Hölder continuous in the sense of local energy norm.

We can now state the theorem as follows:

Theorem. Suppose that $\mu$ (real) does not belong to $\sigma_{p}\left(L_{1}\right) \cup\{0\}$ and let $g$ be any function with bounded support such that $M^{-1} g$ is in $\{I--\widetilde{Q}\} \mathscr{H}_{1}$, where $\widetilde{Q}$ is the projection onto the eigenspace of $L_{1}$ corresponding to non-zero eigen-values. Denote by $u(x, t)$ any solution of the inhomogeneous equation (3) whose initial values $u_{0}(x)$ are also in $\{I-\widetilde{Q}\} \mathscr{H}_{1}$. Then as $t$ tends to infinity $u e^{-i \mu t}$ tends to $w_{-}(\cdot, \sigma)+w$ in the local energy norm, where $w_{\text {- }}$ is the solution of (4) with $\sigma=\mu$ and $f=-M^{-1} g$ satisfying the outgoing radiation condition, and $w$ is some function in $\eta\left(L_{1}\right)$.

Remark 2. The same theorem is already proved by Lax and Phillips in their book [1; Chapter VI, Theorem 2.4] in the case where $M(x)=I$, the space dimension $n$ is odd and $A(x, \xi)$ is nonsingular for each $x \in G$ and $\xi \in \mathbb{R}^{n}-\{0\}$. In [2], however, it is not assumed that $A^{0}(\xi)$ is isotropic.

Proof of the theorem. First we give an expression of the solution of equation (3). Since $L_{1}$ is selfadjoint, it is not difficult to 
see that (3) has a unique solution for each $g$ and $u_{0}$ in $\mathcal{H}_{1}$. Further, with the aid of the Laplace inversion formula, the solution takes the form

$$
\begin{aligned}
u(\cdot, t) & =\frac{1}{2 \pi i} \int_{\alpha-i \infty}^{\infty+i \infty} i e^{\lambda t}\left(L_{1}+i \lambda\right)^{-1} u_{0} d \lambda+ \\
& +\frac{-1}{2 \pi i} \int_{\alpha-i \infty}^{\infty+i \infty} \frac{e^{\lambda t}}{\lambda-i \mu}\left(L_{1}+i \lambda\right)^{-1} M^{-1} g d \lambda \equiv J_{1}+J_{2}
\end{aligned}
$$

for a sufficiently large $\alpha>0$. Note the relations

$$
\begin{gathered}
e^{i L_{1} t}=\int_{-\infty}^{\infty} e^{i \sigma t} d E_{1}(\sigma) \quad(-\infty<t<\infty) \\
\left(L_{1}+i \lambda\right)^{-1}=\int_{-\infty}^{\infty} \frac{1}{\sigma+i \lambda} d E_{1}(\sigma)=\frac{1}{i} \int_{0}^{\infty} e^{-\lambda t} e^{i L_{1} t} d t \quad(\operatorname{Re} \lambda=\alpha>0),
\end{gathered}
$$

where $E_{1}(\sigma)(-\infty<\sigma<\infty)$ denotes the resolution of the identity for $L_{1}$. Then we have

$$
J_{1}=e^{i L_{1}{ }^{t}} u_{0}=\int_{-\infty}^{\infty} e^{i \sigma t} d E_{1}(\sigma) u_{0}
$$

On the other hand, we have

$$
\begin{aligned}
J_{2} & =\frac{-1}{2 \pi i} \int_{\alpha-i \infty}^{\infty+i \infty} \frac{e^{\lambda t}}{\lambda-i \mu} d \lambda \int_{-\infty}^{\infty} \frac{1}{\sigma+i \lambda} d E_{1}(\sigma) M^{-1} g \\
& =\lim _{\varepsilon \rightarrow+0} \int_{-\infty}^{\infty} d E_{1}(\sigma) M^{-1} g \frac{-1}{2 \pi i} \int_{\alpha-i \infty}^{\infty+i \infty} \frac{e^{\lambda t}}{i(\lambda-i \mu)(\lambda+\varepsilon-i \sigma)} d \lambda
\end{aligned}
$$

Since $\frac{e^{\lambda t}}{(\lambda-i \mu)(\lambda+\varepsilon-i \sigma)}$ is an analytic function of the variable $\lambda$ except at the points $\lambda=i \mu$ and $=-\varepsilon+i \sigma(\varepsilon>0)$, using the Cauchy integral theorem and considering the residues for $\lambda=i_{\mu}$ and $=-\varepsilon+i \sigma$, we have for an arbitrary $\alpha^{\prime}>\varepsilon$

$$
\begin{aligned}
\frac{-1}{2 \pi i} \int_{\alpha-i \infty}^{\infty+i \infty} \frac{e^{\lambda t}}{i(\lambda-i \mu)(\lambda+\varepsilon-i \sigma)} d \lambda & =\frac{-1}{2 \pi i} \int_{-\alpha^{\prime}-i \infty}^{-\infty \prime i \infty} \frac{e^{\sigma t}}{i(\lambda-i \mu)(\lambda+\varepsilon-i \sigma)} d \lambda \\
& +\frac{e^{(-\varepsilon+i \sigma) t}}{\sigma-\mu+i \varepsilon}-\frac{e^{i \mu t}}{\sigma-\mu+i \varepsilon} .
\end{aligned}
$$

Summing up, we have the following expression of the solution for $t>0$. 
(6)

$$
\begin{aligned}
u(\cdot, t) & =\int_{-\infty}^{\infty} e^{i \sigma t} d E_{1}(\sigma) u_{0} \\
& +\frac{e^{-\infty \prime t}}{-2 \pi} \int_{-\infty}^{\infty} d E_{1}(\sigma) M^{-1} g \int_{-\infty}^{\infty} \frac{e^{i \tau t}}{\left(\tau-\mu+i \alpha^{\prime}\right)\left(\tau-\sigma+i \alpha^{\prime}\right)} d \tau \\
& +\lim _{\varepsilon \rightarrow 10} \int_{-\infty}^{\infty} \frac{e^{(-\varepsilon+i \sigma) t}-e^{i \mu t}}{\sigma-\mu+i \varepsilon} d E_{1}(\sigma) M^{-1} g,
\end{aligned}
$$

where $\alpha^{\prime}$ is any positive constant and the limit is taken in the strong sense in $\mathscr{H}_{1}$.

It is obvious that the second term of the right member tends as $t \rightarrow \infty$ to zero in the sense of $\mathscr{H}_{1}$-norm. Thus our problem is to know how the first and third terms behave for $t$ large. For this purpose we use relation (5) which holds for any $f$ in $\mathscr{H}_{1}$ with bounded support.

The first term can be divided as follows:

$$
\begin{aligned}
\int_{-\infty}^{\infty} e^{i \sigma t} d E_{1}(\sigma) u_{0} & =\left(\int_{-\infty}^{-r}+\int_{r}^{\infty}\right) e^{i \sigma t} d E_{1}(\sigma) u_{0}+\sum_{l=0}^{N_{r}} \int_{e_{l}} e^{i \sigma t} d E_{1}(\sigma) u_{0} \\
& +\int_{[-r, r]-v_{e_{l}}} e^{i \sigma t} d E_{1}(\sigma) u_{0}
\end{aligned}
$$

where $r>0$ is chosen sufficiently large so that $\left\|E_{1}(-r) u_{0}\right\|_{1}+$ $\left\|\left\{I-E_{1}(r)\right\} u_{0}\right\|_{1}<\varepsilon / 5$ for given any $\varepsilon>0, e_{0}$ is a neighborhood of the origin and $e_{l}\left(l=1,2, \cdots, N_{r}\right)$ are neighborhoods of non-zero eigenvalues $\sigma_{l}$ of $L_{1}$ such that $\left|\sigma_{l}\right|<r$. Since we assumed that $u_{0}$ is in $(I-\widetilde{Q}) \mathscr{H}_{1}$, it follows that

$$
\sum_{l=1}^{N_{r}} \int_{e_{l}} d\left\|E_{1}(\sigma) u_{0}\right\|_{1}<\varepsilon / 5
$$

if we choose $e_{l}$ sufficiently small. Further, if we put $w_{1}=\left\{E_{1}(+0)\right.$ $\left.-E_{1}(-0)\right\} u_{0}$, then $w_{1} \in \mathscr{R}\left(L_{1}\right)$ and it follows that

$$
\left\|\int_{e_{0}} e^{i \sigma t} d E_{1}(\sigma) u_{0}-w_{1}\right\|_{1}<\varepsilon / 5
$$

for a sufficiently small $e_{0}$. On the other hand, there exists a function $f$ in $\left[C_{0}^{\infty}(G)\right]^{m}$ such that $\left\|u_{0}-f\right\|_{1}<\varepsilon / 5$. For $E_{1}(\sigma) f$, we can use relation (5) to obtain

$$
\int_{[-r, r]-v_{e_{l}}} e^{i \sigma t} d E_{1}(\sigma) f=\int_{[-r, r]-v_{l}} e^{i \sigma t}\left\{v_{\vdash}(\cdot, \sigma)-v_{-}(\cdot, \sigma)\right\} d \sigma .
$$


Since every local energy norm of $v_{+}(\cdot, \sigma)-v_{-}(\cdot, \sigma)$ is bounded continuous in $\sigma$, it follows from the Riemann-Lebesgue theorem that for any bounded subdomain $G^{\prime}$ of $G$

$$
\left\|\int_{[-r, r]-u_{e}} e^{i \sigma t} d E_{1}(\sigma) f\right\|_{1, G^{\prime}}<\varepsilon / 5
$$

for sufficiently large $t$. Summing up we conclude that $\int_{-\infty}^{\infty} e^{i \sigma t} d E_{1}(\sigma) u_{0}$ tends to $w_{1}$ as $t \rightarrow \infty$ in the sense of the local energy norm.

Next we consider the third term of the right member of (6).

$$
\begin{aligned}
& \lim _{\varepsilon \rightarrow+0}\left\{\int_{-\infty}^{\infty} \frac{e^{(-\varepsilon+i \sigma) t}}{\sigma-\mu+i \varepsilon} d E_{1}(\sigma) M^{-1} g-e^{i \mu t} \int_{-\infty}^{\infty} \frac{1}{\sigma-\mu+i \varepsilon} d E_{1}(\sigma) M^{-1} g\right\} \\
& \quad=\lim _{\varepsilon \rightarrow+0}\left\{\int_{-\infty}^{\infty} \frac{e^{(-\varepsilon+i \sigma) t}}{\sigma-\mu+i \varepsilon} d E_{1}(\sigma) M^{-1} g-e^{i \mu t}\left(L_{1}-\mu+i \varepsilon\right)^{-1} M^{-1} g\right\} \\
& \quad=J-e^{i \mu t} w_{-}(\cdot, \mu),
\end{aligned}
$$

where

$$
J=\lim _{\varepsilon \rightarrow+0} \int_{-\infty}^{\infty} \frac{e^{\left(-\varepsilon_{1} i \sigma\right) t}}{\sigma-\mu+i \varepsilon} d E_{1}(\sigma) M^{-1} g
$$

and the limit is taken in the sense of local energy norm. By the same reasoning as above, we see that every local energy norm of

$$
J-\frac{w_{2}}{\mu}-\lim _{\varepsilon \rightarrow+0} \int_{[-r, r]-\cup e} \frac{e^{(-\varepsilon|i \sigma| t}}{\sigma-\mu+i \varepsilon} \varphi(\cdot, \sigma) d \sigma
$$

becomes as small as we wish if we choose $r$ sufficiently large and $e_{l}$ sufficiently small, where $w_{2}=\left\{E_{1}(+0)-E_{1}(-0)\right\} M^{-1} g$ and $\varphi=w_{\dashv}-w_{-}$, taking into account that $\mu$ (we can assume that $-r<\mu<r$ ) is not in $\cup e_{l}$. On the other hand, if we note that

$$
\lim _{t \rightarrow \infty} \lim _{\varepsilon \rightarrow+0} \int_{e} \frac{e^{(-\varepsilon+i \sigma) t}}{\sigma+i \varepsilon} d \sigma=0
$$

for any interval $e$ which includes the origin, then we have

$$
\begin{aligned}
& \lim _{t \rightarrow \infty} \lim _{\varepsilon \rightarrow+0} \int_{[-r, r]-v e_{l}} \frac{e^{(-\varepsilon+i \sigma) t}}{\sigma-\mu+i \varepsilon} \varphi(\cdot, \sigma) d \sigma \\
& \quad=\lim _{t \rightarrow \infty} \int_{[-r, r]-v e_{l}-\tilde{e}} \frac{\varphi(\cdot, \sigma)}{\sigma-\mu} e^{i \sigma t} d \sigma+\lim _{t \rightarrow \infty} e^{i \mu t} \int_{e} \frac{\varphi(\cdot, \sigma+\mu)-\varphi(\cdot, \mu)}{\sigma} e^{i \sigma t} d \sigma,
\end{aligned}
$$

where $\tilde{e}$ is an interval in $[-r, r]-\cup e_{l}$ which contains the point $\mu$ and $e=\{\sigma-\mu ; \sigma \in \hat{\rho}\}$. Noting that $\varphi(\cdot, \sigma)$ is Hölder continuous in 
$\sigma$, we can use again the Riemann-Lebesgue theorem to show that $J$ tends to $w_{2} / \mu$ as $t \rightarrow \infty$. Thus we see that the third term of the right member of (6) tends to $e^{i \mu t} w_{-}+w_{2} / \mu$ as $t \rightarrow \infty$ in the sense of the local energy norm. The proof is now complete.

Remark 3. As we see in the proof, the Hölder continuity of $\frac{d}{d \sigma} E_{1}(\sigma) f$ in $\sigma$ is used only to estimate the term containing $g(x)$. Thus, if $g(x) \equiv 0$, we do not require that $A(x, \xi)$ is non-singular (i.e., we can replace (III) by assumption (iv) in [2]) to derive the theorem. The above theorem asserts the local energy decay of solutions if $g(x) \equiv 0$.

Remark 4. The local energy decay of solutions has been essentially proved in [2] by Theorem 6.2 since obviously $e^{i L_{0}{ }^{t}} f^{-}$tends to zero as $t \rightarrow \infty$ in the local energy norm, where $L_{0}=\frac{1}{i} \sum_{j=1}^{n} A_{j} \frac{\partial}{\partial x_{j}}$ is the unperturbed operator acting in $\mathscr{H}_{0}=\left[L^{2}\left(\mathbb{R}^{n}\right)\right]^{m}$ and $f \in \mathscr{H}_{0} \fallingdotseq \cdot \eta\left(I_{\nu_{0}}\right)$ is determined uniquely from the initial data $u_{0}$.

\section{References}

[1] Lax, P. D., and R. S. Phillips, Scattering Theory, Academic Press, New York, 1967.

[2] Mochizuhi, K., Spectral and scattering theory for symmetric hyperbolic sjstems in an exlerior domain, Publ. RIMS Kyoto Univ., rliis issue. 
